Mathematical Modelling and Analysis

Volume 22 Number 4, July 2017, 514-527

https://doi.org/10.3846/13926292.2017.1329756

(C) Vilnius Gediminas Technical University, 2017
Publisher: Taylor\&Francis and VGTU

http://www.tandfonline.com/TMMA

ISSN: $1392-6292$

eISSN: $1648-3510$

\title{
Cubic Spline Histopolation*
}

\author{
Evely Kirsiaed ${ }^{a}$, Peeter $\mathrm{Oja}^{a}$ and Gul Wali Shah ${ }^{a}$ \\ ${ }^{a}$ University of Tartu, Institute of Mathematics and Statistics \\ J. Liivi 2, Tartu \\ E-mail(corresp.): evely.kirsiaed@ut.ee \\ E-mail: peeter.oja@ut.ee \\ E-mail: gulwali@ut.ee
}

Received September 23, 2016; revised May 8, 2017; published online July 15, 2017

\begin{abstract}
Cubic spline histopolation with arbitrary placement of histogram knots and spline knots between them is studied. Classical boundary conditions are used. Histopolating spline is represented with the help of second moments and particular integrals. The systems determining these parameters are investigated in different cases where diagonal dominance in matrices takes place or may be absent.
\end{abstract}

Keywords: histopolation, cubic spline, existence of histopolant.

AMS Subject Classification: 65D07; 41A15.

\section{Introduction}

The histopolation problem is more practical than the interpolation problem as, e.g., the statistical information is rather given in form of histograms. On the other hand, for any given histopolation problem an equivalent interpolation problem could be formulated and the derivative of the interpolant is, in fact, the histopolant [19]. We treat in this paper the histopolation problem with cubic splines. However, instead of that, it is possible to solve the corresponding interpolation problem with quartic splines and afterwards calculate its derivative. This additional step in practice accompanies with additional (at least round-off) errors at calculations. Because of that it is preferable to have direct algorithms for finding the cubic spline histopolant. In other words, instead of implicit theory via quartic spline interpolation we develop explicit theory of cubic spline histopolation. We consider most common boundary conditions like given values of the spline and its first and second derivatives in endpoints of given interval.

A wide class at solving differential and integral equations is projection methods. The collocation method is the interpolation projection method and the

\footnotetext{
* The research was supported by institutional research funding IUT20-57 of the Estonian Ministry of Education and Research.
} 
subdomain method is the histopolating projection method. The application of these methods requires detailed description of interpolation and histopolation processes, respectively. It is remarkable that, for the boundary value problem with second order linear differential equation, on uniform mesh, the collocation with cubic splines has the rate $\mathcal{O}\left(h^{2}\right)[10,17,18]$ but the subdomain method has $\mathcal{O}\left(h^{4}\right)[15,18]$. The subdomain method is very natural if, e.g., the free term function in differential equation is given approximately via mean values on subintervals. The same idea works well in case of Volterra integral equations [5]. These circumstances are a great motivation to give a special attention to histopolation problem with cubic splines.

The histopolation with splines is studied in many papers under different names like area matching interpolation $[2,3,6]$, interpolation in the mean $[4,6$, 20], interpolation of mean values [11], histospline [20]. The spline histopolation on biinfinite knot sequence is treated in [20]. There are several papers by quartic spline interpolation, e.g., $[11,12,13]$ but the interpolation problem which is equivalent to the histopolation with cubic splines is not treated in them in such extent as we do in current paper.

We confirm with numerical examples the known fact that the cubic spline interpolant and also the cubic spline histopolant (which is, in fact, an interpolant in intermediate points) do not preserve geometrical properties. One way to overcome this disadvantage is to use rational or combined splines $[7,8]$. But rational interpolating or histopolating splines do not exist for any data $[7,16]$, cubic spline interpolants or histopolants exist always. Another idea to preserve geometrical properties is to add some auxiliary spline knots (see, e.g., [14] to preserve monotonicity). In [8] monotonicity is preserved without auxiliary knots by using quadratic and rational spline pieces. The convexity preserving combined spline theory similar to [8] should use cubic spline histopolation which we develop in this paper.

\section{The histopolation problem}

Let $x_{i}$ be given points on an interval $[a, b]$ such that $a=x_{0}<x_{1}<\ldots<x_{n}=b$ and let $z_{i}, i=1, \ldots, n$, be given real numbers (histogram heights). Denote $h_{i}=x_{i}-x_{i-1}, i=1, \ldots, n$. We consider the problem of finding a function $S:[a, b] \rightarrow \mathbb{R}$ such that

$$
\int_{x_{i-1}}^{x_{i}} S(x) d x=z_{i} h_{i}, \quad i=1, \ldots, n
$$

and $S$ is a cubic spline from the class $C^{2}$. Conditions (2.1) are called histopolation conditions.

Since a cubic spline with $\operatorname{knots} x_{i}, i=0, \ldots, n$, has $n+3$ free parameters (dimension of the cubic spline space is $n+3$ ), and this could not be well combined with (2.1), we choose cubic spline knots as

$$
\xi_{1}=x_{0}, \quad \xi_{i} \in\left(x_{i-1}, x_{i}\right), i=2, \ldots, n-1, \quad \xi_{n}=x_{n} .
$$


Then the cubic spline has $n+2$ free parameters. We add to the histopolation conditions two boundary conditions from

$$
\begin{aligned}
& S(a)=\alpha, \quad S^{\prime}(a)=\alpha, \quad S^{\prime \prime}(a)=\alpha, \\
& S(b)=\beta, \quad S^{\prime}(b)=\beta, \quad S^{\prime \prime}(b)=\beta
\end{aligned}
$$

at different endpoints $a$ and $b$.

\section{Representation of the histopolant}

Several representations of cubic spline could be considered, but the one which uses second moments and particular integrals is appropriate. Thus, on the interval $\left[\xi_{i}, \xi_{i+1}\right]$ we use four parameters to represent the spline:

$$
M_{i}=S^{\prime \prime}\left(\xi_{i}\right), \quad M_{i+1}=S^{\prime \prime}\left(\xi_{i+1}\right), \quad \lambda_{i}=\int_{\xi_{i}}^{x_{i}} S(x) d x, \quad \rho_{i}=\int_{x_{i}}^{\xi_{i+1}} S(x) d x .
$$

Denote $\varepsilon_{i}=x_{i}-\xi_{i}, \eta_{i}=\xi_{i+1}-x_{i}, \delta_{i}=\varepsilon_{i}+\eta_{i}, i=1, \ldots, n-1$ (see Figure 1).

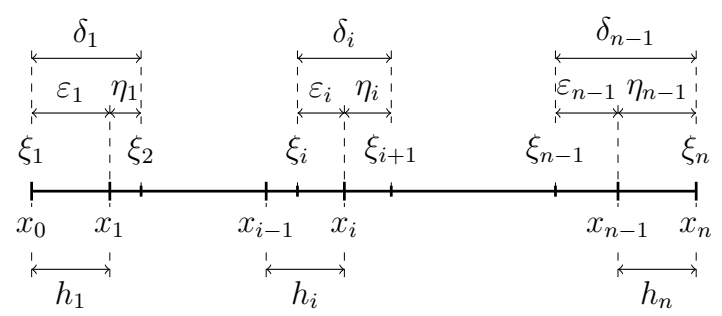

Figure 1. Additional parameters.

Then the spline could be written as

$$
\begin{array}{ll}
S(x)=a_{i}+b_{i}\left(x-x_{i}\right)+c_{i}\left(x-x_{i}\right)^{2}+d_{i}\left(x-x_{i}\right)^{3}, & x \in\left[\xi_{i}, \xi_{i+1}\right], \\
& i=1, \ldots, n-1,
\end{array}
$$

where

$$
\begin{aligned}
c_{i} & =\frac{M_{i} \eta_{i}+M_{i+1} \varepsilon_{i}}{2 \delta_{i}}, \quad d_{i}=\frac{M_{i+1}-M_{i}}{6 \delta_{i}}, \\
a_{i} & =\frac{1}{\delta_{i}}\left(\lambda_{i} \frac{\eta_{i}}{\varepsilon_{i}}+\rho_{i} \frac{\varepsilon_{i}}{\eta_{i}}\right)-\frac{c_{i}}{3} \varepsilon_{i} \eta_{i}-\frac{d_{i}}{4}\left(\eta_{i}-\varepsilon_{i}\right) \varepsilon_{i} \eta_{i}, \\
b_{i} & =\frac{2}{\delta_{i}}\left(\frac{\rho_{i}}{\eta_{i}}-\frac{\lambda_{i}}{\varepsilon_{i}}\right)-2\left(\frac{c_{i}}{3}\left(\eta_{i}-\varepsilon_{i}\right)+\frac{d_{i}}{4}\left(\eta_{i}^{2}-\eta_{i} \varepsilon_{i}+\varepsilon_{i}^{2}\right)\right) .
\end{aligned}
$$

To determine the parameters $M_{i}, i=1, \ldots, n, \lambda_{i}, \rho_{i}, i=1, \ldots, n-1$, we use smoothness conditions

$$
S\left(\xi_{i}-0\right)=S\left(\xi_{i}+0\right), \quad i=2, \ldots, n-1,
$$




$$
S^{\prime}\left(\xi_{i}-0\right)=S^{\prime}\left(\xi_{i}+0\right), \quad i=2, \ldots, n-1,
$$

histopolation conditions

$$
\rho_{i-1}+\lambda_{i}=z_{i} h_{i}, \quad i=1, \ldots, n
$$

with $\rho_{0}=0, \lambda_{n}=0$, and two boundary conditions. Equations (3.2) and (3.3) take the form, respectively,

$$
\begin{aligned}
-\frac{\eta_{i-1}}{\delta_{i-1} \varepsilon_{i-1}} & \lambda_{i-1}+\frac{1}{\delta_{i-1}}\left(2+\frac{\varepsilon_{i-1}}{\eta_{i-1}}\right) \rho_{i-1}-\frac{1}{\delta_{i}}\left(2+\frac{\eta_{i}}{\varepsilon_{i}}\right) \lambda_{i}+\frac{\varepsilon_{i}}{\delta_{i} \eta_{i}} \rho_{i} \\
= & \frac{1}{24}\left[-\eta_{i-1}\left(\varepsilon_{i-1}+2 \eta_{i-1}\right) M_{i-1}\right. \\
& +\left(-\eta_{i-1}\left(3 \varepsilon_{i-1}+2 \eta_{i-1}\right)+\varepsilon_{i}\left(2 \varepsilon_{i}+3 \eta_{i}\right)\right) M_{i} \\
& \left.+\varepsilon_{i}\left(2 \varepsilon_{i}+\eta_{i}\right) M_{i+1}\right], \quad i=2, \ldots, n-1, \\
-\frac{1}{\delta_{i-1} \varepsilon_{i-1}} & \lambda_{i-1}+\frac{1}{\delta_{i-1} \eta_{i-1}} \rho_{i-1}+\frac{1}{\delta_{i} \varepsilon_{i}} \lambda_{i}-\frac{1}{\delta_{i} \eta_{i}} \rho_{i} \\
= & -\frac{1}{24}\left[\frac{\delta_{i-1}^{2}+\eta_{i-1} \delta_{i-1}+\eta_{i-1}^{2}}{\delta_{i-1}} M_{i-1}\right. \\
& +\left(\frac{3 \delta_{i-1}^{2}+2 \eta_{i-1} \delta_{i-1}+\eta_{i-1} \varepsilon_{i-1}}{\delta_{i-1}}+\frac{3 \delta_{i}^{2}+2 \varepsilon_{i} \delta_{i}+\varepsilon_{i} \eta_{i}}{\delta_{i}}\right) M_{i} \\
& \left.+\frac{\delta_{i}^{2}+\varepsilon_{i} \delta_{i}+\varepsilon_{i}^{2}}{\delta_{i}} M_{i+1}\right], \quad i=2, \ldots, n-1 .
\end{aligned}
$$

Observe that these equations are linear (homogeneous) with respect to the unknowns $\lambda_{i-1}, \rho_{i-1}, \lambda_{i}, \rho_{i}, M_{i-1}, M_{i}, M_{i+1}$.

\section{Systems defining spline parameters}

In total, we have to determine $3 n-2$ unknowns $M_{1}, \ldots, M_{n}, \lambda_{1}, \ldots, \lambda_{n-1}$, $\rho_{1}, \ldots, \rho_{n-1}$ from the system of $3 n-2$ equations: (3.4), (3.5), (3.6) and two boundary conditions. This system is of undetermined form to study. We take 9 equations $(3.4, i-1),(3.5, i-1),(3.6, i-1),(3.4, i),(3.5, i),(3.6, i),(3.4, i+1)$, $(3.5, i+1),(3.6, i+1)$ containing eight unknowns $\lambda_{i-2}, \rho_{i-2}, \lambda_{i-1}, \rho_{i-1}, \lambda_{i}, \rho_{i}$, $\lambda_{i+1}, \rho_{i+1}$. These $\lambda_{j}, \rho_{j}$ could be eliminated by using the linear combination of equations with coefficients indicated below:

$$
\begin{aligned}
& (3.4, i-1) \quad-\frac{h_{i}+h_{i+1}}{h_{i-1}}, \quad(3.5, i-1) \quad \frac{h_{i}+h_{i+1}}{h_{i-1}} \eta_{i-2}, \\
& (3.6, i-1) \quad-\frac{h_{i}+h_{i+1}}{h_{i-1}} \eta_{i-2}^{2}, \quad(3.4, i) \frac{h_{i-1}+2 h_{i}+h_{i+1}}{h_{i}}, \\
& (3.5, i) \frac{\varepsilon_{i}\left(h_{i}+h_{i+1}\right)-\eta_{i-1}\left(h_{i-1}+h_{i}\right)}{h_{i}}, \\
& (3.6, i) \frac{\varepsilon_{i}^{2}\left(h_{i}+h_{i+1}\right)+\eta_{i-1}^{2}\left(h_{i-1}+h_{i}\right)}{h_{i}}-\left(h_{i-1}+h_{i}\right)\left(h_{i}+h_{i+1}\right),
\end{aligned}
$$




$$
\begin{aligned}
& (3.4, i+1) \quad-\frac{h_{i-1}+h_{i}}{h_{i+1}}, \quad(3.5, i+1)-\frac{h_{i-1}+h_{i}}{h_{i+1}} \varepsilon_{i+1}, \\
& (3.6, i+1) \quad-\frac{h_{i-1}+h_{i}}{h_{i+1}} \varepsilon_{i+1}^{2} .
\end{aligned}
$$

For $i=3, \ldots, n-2$ we obtain the equation

$$
c_{i, i-2} M_{i-2}+c_{i, i-1} M_{i-1}+c_{i i} M_{i}+c_{i, i+1} M_{i+1}+c_{i, i+2} M_{i+2}=D_{i},
$$

where

$$
\begin{aligned}
& D_{i}=\left(h_{i}+h_{i+1}\right) z_{i-1}-\left(h_{i-1}+2 h_{i}+h_{i+1}\right) z_{i}+\left(h_{i-1}+h_{i}\right) z_{i+1}, \\
& c_{i, i-2}=\frac{1}{24} \frac{\eta_{i-2}^{4}\left(h_{i}+h_{i+1}\right)}{\delta_{i-2} h_{i-1}}, \\
& c_{i, i-1}=\frac{1}{24}\left(\left(\eta_{i-2}\left(3 \eta_{i-2}+2 \varepsilon_{i-1}+3 \eta_{i-1}\right)+\left(\varepsilon_{i-1}+2 \eta_{i-1}\right)\left(h_{i-1}+\eta_{i-1}\right)\right.\right. \\
& \left.+\frac{\eta_{i-2}^{2}}{h_{i-1}}\left(\frac{\varepsilon_{i-2} \eta_{i-2}}{\delta_{i-2}}+\frac{\varepsilon_{i-1} \eta_{i-1}}{\delta_{i-1}}\right)\right)\left(h_{i}+h_{i+1}\right) \\
& \left.+\frac{\eta_{i-1}^{3} \varepsilon_{i}\left(h_{i-1}+h_{i}+h_{i+1}\right)}{\delta_{i-1} h_{i}}+\frac{\eta_{i-1}^{2}\left(h_{i-1}+\eta_{i-1}\right)\left(\varepsilon_{i}+h_{i+1}\right)}{\delta_{i-1}}\right), \\
& c_{i i}=\frac{1}{24}\left(\left(\eta_{i-2}\left(2 \varepsilon_{i-1}+\eta_{i-1}\right)+\left(3 \varepsilon_{i-1}+2 \eta_{i-1}\right)\left(h_{i-1}+\eta_{i-1}\right)\right.\right. \\
& \left.+\frac{\eta_{i-2}^{2} \varepsilon_{i-1}^{2}}{\delta_{i-1} h_{i-1}}\right)\left(h_{i}+h_{i+1}\right) \\
& +\left(\varepsilon_{i+1}\left(\varepsilon_{i}+2 \eta_{i}\right)+\left(2 \varepsilon_{i}+3 \eta_{i}\right)\left(\varepsilon_{i}+h_{i+1}\right)+\frac{\eta_{i}^{2} \varepsilon_{i+1}^{2}}{\delta_{i} h_{i+1}}\right)\left(h_{i-1}+h_{i}\right) \\
& +\left(\left(3+\frac{\varepsilon_{i-1}}{\delta_{i-1}}\right) \eta_{i-1}+\left(3+\frac{\eta_{i}}{\delta_{i}}\right) \varepsilon_{i}\right)\left(\left(h_{i-1}+\eta_{i-1}\right)\left(\varepsilon_{i}+h_{i+1}\right)\right. \\
& \left.\left.+\eta_{i-1} \varepsilon_{i} \frac{h_{i-1}+h_{i}+h_{i+1}}{h_{i}}\right)\right) \text {, } \\
& c_{i, i+1}=\frac{1}{24}\left(\left(\varepsilon_{i+1}\left(3 \varepsilon_{i}+2 \eta_{i}+3 \varepsilon_{i+1}\right)+\left(2 \varepsilon_{i}+\eta_{i}\right)\left(\varepsilon_{i}+h_{i+1}\right)\right.\right. \\
& \left.+\frac{\varepsilon_{i+1}^{2}}{h_{i+1}}\left(\frac{\varepsilon_{i} \eta_{i}}{\delta_{i}}+\frac{\varepsilon_{i+1} \eta_{i+1}}{\delta_{i+1}}\right)\right)\left(h_{i-1}+h_{i}\right) \\
& \left.+\frac{\eta_{i-1} \varepsilon_{i}^{3}\left(h_{i-1}+h_{i}+h_{i+1}\right)}{\delta_{i} h_{i}}+\frac{\varepsilon_{i}^{2}\left(h_{i-1}+\eta_{i-1}\right)\left(\varepsilon_{i}+h_{i+1}\right)}{\delta_{i}}\right), \\
& c_{i, i+2}=\frac{1}{24} \frac{\varepsilon_{i+1}^{4}\left(h_{i-1}+h_{i}\right)}{\delta_{i+1} h_{i+1}} .
\end{aligned}
$$

Let us notice certain symmetry in equation (4.1). There are symmetric pairs of parameters: $h_{i-1} \leftrightarrow h_{i+1}, \delta_{i-2} \leftrightarrow \delta_{i+1}, \delta_{i-1} \leftrightarrow \delta_{i}, \eta_{i-2} \leftrightarrow \varepsilon_{i+1}, \varepsilon_{i-1} \leftrightarrow \eta_{i}$, 
$\eta_{i-1} \leftrightarrow \varepsilon_{i}$. Then we see the symmetry between $c_{i, i-2}$ and $c_{i, i+2}, c_{i, i-1}$ and $c_{i, i+1}$, inside $c_{i i}$. However, all coefficients (4.3)-(4.7) are positive.

In case of $i=2$ we take seven equations $(3.4,1),(3.4,2),(3.5,2),(3.6,2)$, $(3.4,3),(3.5,3),(3.6,3)$ to eliminate six unknowns $\lambda_{1}, \rho_{1}, \lambda_{2}, \rho_{2}, \lambda_{3}, \rho_{3}$. The coefficients of the appropriate linear combination are as in general case. This leads to the equation

$$
c_{21} M_{1}+c_{22} M_{2}+c_{23} M_{3}+c_{24} M_{4}=D_{2},
$$

where $D_{2}$ is determined by (4.2), $c_{23}$ and $c_{24}$ by (4.6) and (4.7), respectively. There is certain difference in $c_{21}$ and $c_{22}$ compared to (4.4) and (4.5), but they could be calculated similarly to the general case taking into account also the configuration of the intervals near the endpoint $a$.

Similar situation takes place in case of $i=n-1$.

The simplest boundary equation here is $S^{\prime \prime}(a)=\alpha$ or $M_{1}=\alpha$. The other possible boundary conditions, e.g., $S(a)=\alpha$ and $S^{\prime}(a)=\alpha$, require the calculation of $S\left(\xi_{1}+0\right)$ and $S^{\prime}\left(\xi_{1}+0\right)$ as it was done at transformation of (3.2) and (3.3). This should be followed by the elimination of appearing parameters $\lambda_{j}$, $\rho_{j}$. Both cases give us the equation

$$
c_{11} M_{1}+c_{12} M_{2}+c_{13} M_{3}=D_{1}
$$

with certain expression $D_{1}$ depending on $\alpha$ and given histogram parameters. However, (4.8) includes $M_{1}=\alpha$.

The boundary conditions at the endpoint $b$ could be treated similarly.

Thus, the spline parameters $M_{1}, \ldots, M_{n}$ are determined by the five-diagonal system

$$
\left\{\begin{array}{l}
c_{11} M_{1}+c_{12} M_{2}+c_{13} M_{3}=D_{1}, \\
c_{21} M_{1}+c_{22} M_{2}+c_{23} M_{3}+c_{24} M_{4}=D_{2}, \\
c_{i, i-2} M_{i-2}+c_{i, i-1} M_{i-1}+c_{i i} M_{i}+c_{i, i+1} M_{i+1}+c_{i, i+2} M_{i+2}=D_{i}, \\
i=3, \ldots, n-2, \\
c_{n-1, n-3} M_{n-3}+c_{n-1, n-2} M_{n-2}+c_{n-1, n-1} M_{n-1}+c_{n-1, n} M_{n} \\
=D_{n-1}, \\
c_{n, n-2} M_{n-2}+c_{n, n-1} M_{n-1}+c_{n n} M_{n}=D_{n} .
\end{array}\right.
$$

Solving this, the system consisting of all equations (3.5), (3.6) allows to determine the parameters $\lambda_{j}, \rho_{j}$. Its unique solvability is shown in [9]. Note that the values $\lambda_{1}$ and $\rho_{n-1}$ are known due to the histopolation conditions $(3.4,1)$ and $(3.4, n)$. We discuss the solvability of (4.9) in next section.

\section{Existence and uniqueness of the solution}

It is clear that the unique solvability of system (4.9) is equivalent to the existence of unique solution to the histopolation problem. Let us start with particular cases. 
Consider the case of spline knots as $\xi_{i}=\left(x_{i-1}+x_{i}\right) / 2, i=2, \ldots, n-1$. Then $\eta_{i-1}=\varepsilon_{i}=h_{i} / 2, i=2, \ldots, n-1, \varepsilon_{1}=h_{1}, \eta_{n-1}=h_{n}$. The coefficients in (4.1) are (we write them also keeping symmetrical structure)

$$
\begin{aligned}
c_{i, i-2}= & \frac{1}{192}\left(h_{i}+h_{i+1}\right) \frac{h_{i-1}^{3}}{h_{i-2}+h_{i-1}}, \\
c_{i, i-1}= & \frac{1}{192}\left(\left(h_{i}+h_{i+1}\right)\left(14 h_{i-1}^{2}+17 h_{i-1} h_{i}+6 h_{i}^{2}+\frac{h_{i-2} h_{i-1}^{2}}{h_{i-2}+h_{i-1}}\right)+h_{i}^{2} h_{i+1}\right), \\
c_{i i}= & \frac{1}{192}\left(\left(h_{i}+h_{i+1}\right)\left(17 h_{i-1}^{2}+30 h_{i-1} h_{i}+10 h_{i}^{2}\right)\right. \\
& \left.+\left(h_{i-1}+h_{i}\right)\left(10 h_{i}^{2}+30 h_{i} h_{i+1}+17 h_{i+1}^{2}\right)+2 h_{i-1} h_{i} h_{i+1}\right), \\
c_{i, i+1}= & \frac{1}{192}\left(\left(h_{i-1}+h_{i}\right)\left(6 h_{i}^{2}+17 h_{i} h_{i+1}+14 h_{i+1}^{2}+\frac{h_{i+1}^{2} h_{i+2}}{h_{i+1}+h_{i+2}}\right)+h_{i-1} h_{i}^{2}\right), \\
c_{i, i+2}= & \frac{1}{192}\left(h_{i-1}+h_{i}\right) \frac{h_{i+1}^{3}}{h_{i+1}+h_{i+2}} .
\end{aligned}
$$

We see here the diagonal dominance in rows as

$$
\begin{aligned}
c_{i i}-\left(c_{i, i-2}+c_{i, i-1}+c_{i, i+1}+c_{i, i+2}\right) & \\
= & \frac{1}{192}\left(\left(h_{i}+h_{i+1}\right)\left(2 h_{i-1}^{2}+13 h_{i-1} h_{i}+3 h_{i}^{2}\right)\right. \\
\quad & \left.\quad\left(h_{i-1}+h_{i}\right)\left(3 h_{i}^{2}+13 h_{i} h_{i+1}+2 h_{i+1}^{2}\right)+2 h_{i}^{3}+2 h_{i-1} h_{i} h_{i+1}\right) .
\end{aligned}
$$

Similar calculations give the diagonal dominance in near-boundary equations which yields the unique solvability of (4.9) in this case.

In case of uniform mesh with $h_{i}=h, i=1, \ldots, n$, and $\xi_{i}=\left(x_{i-1}+x_{i}\right) / 2$, $i=2, \ldots, n-1$, the interior equations of (4.9) are

$$
\left\{\begin{array}{l}
\frac{h^{3}}{192}\left(52 M_{1}+255 M_{2}+76 M_{3}+M_{4}\right)=D_{2}, \\
\frac{h^{3}}{576}\left(2 M_{1}+229 M_{2}+690 M_{3}+228 M_{4}+3 M_{5}\right)=D_{3} \\
\frac{h^{3}}{192}\left(M_{i-2}+76 M_{i-1}+230 M_{i}+76 M_{i+1}+M_{i+2}\right)=D_{i}, \quad i=4, \ldots, n-3, \\
\frac{h^{3}}{576}\left(3 M_{n-4}+228 M_{n-3}+690 M_{n-2}+229 M_{n-1}+2 M_{n}\right)=D_{n-2}, \\
\frac{h^{3}}{192}\left(M_{n-3}+76 M_{n-2}+255 M_{n-1}+52 M_{n}\right)=D_{n-1} .
\end{array}\right.
$$

The boundary condition $S(a)=\alpha$ gives the equation

$$
\frac{1}{1152}\left(386 M_{1}+379 M_{2}+3 M_{3}\right)=\frac{1}{h^{2}}\left(2 \alpha-3 z_{1}+z_{2}\right),
$$

$S(b)=\beta$ gives

$$
\frac{1}{1152}\left(3 M_{n-2}+379 M_{n-1}+386 M_{n}\right)=\frac{1}{h^{2}}\left(z_{n-1}-3 z_{n}+2 \beta\right),
$$


$S^{\prime}(a)=\alpha$ leads to

$$
\frac{1}{1152}\left(706 M_{1}+443 M_{2}+3 M_{3}\right)=\frac{1}{h^{2}}\left(z_{2}-z_{1}-\alpha h\right)
$$

$S^{\prime}(b)=\beta$ to

$$
\frac{1}{1152}\left(3 M_{n-2}+443 M_{n-1}+706 M_{n}\right)=\frac{1}{h^{2}}\left(h \beta+z_{n-1}-z_{n}\right) .
$$

In general case, there may be no diagonal dominance in equations (4.1). Let us prove that. Consider in coefficients (4.3)-(4.7) the situation where $\eta_{i-2}=$ const $>0$ and other used parameters $\eta_{j}, \varepsilon_{j}$ are equal to $\varepsilon \rightarrow 0$. Then $c_{i, i-2}$ is of order $\eta_{i-2}^{2} \varepsilon$ but $c_{i i}$ has the order $\eta_{i-2} \varepsilon^{2}$.

The unique solvability of system (4.9) follows from the next result.

Proposition 1. The histopolation problem posed in Section 2 has the unique solution.

Proof. It is sufficient to prove that the corresponding homogeneous problem has only trivial solution. Suppose a cubic spline $S$ satisfies

$$
\int_{x_{i-1}}^{x_{i}} S(x) d x=0, \quad i=1, \ldots, n
$$

and two of the boundary conditions $S(a)=0, S(b)=0, S^{\prime}(a)=0, S^{\prime}(b)=0$, $S^{\prime \prime}(a)=0, S^{\prime \prime}(b)=0$ at different endpoints $a$ and $b$. By (5.1) it exists $\eta_{i} \in\left(x_{i-1}, x_{i}\right)$ such that $S\left(\eta_{i}\right)=0, i=1, \ldots, n$.

If $S(a)=S(b)=0$ then there are $\bar{\eta}_{i} \in\left(\eta_{i-1}, \eta_{i}\right), i=2, \ldots, n, \bar{\eta}_{1} \in\left(a, \eta_{1}\right)$, $\bar{\eta}_{n+1} \in\left(\eta_{n}, b\right)$ such that $S^{\prime}\left(\bar{\eta}_{i}\right)=0, i=1, \ldots, n+1$. Therefore, there are $\overline{\bar{\eta}}_{i} \in\left(\bar{\eta}_{i}, \bar{\eta}_{i+1}\right), i=1, \ldots, n$, such that $S^{\prime \prime}\left(\overline{\bar{\eta}}_{i}\right)=0$. Consequently, an interval $\left[\xi_{k}, \xi_{k+1}\right]$ contains two (distinct) zeros of $S^{\prime \prime}$ which means that $S^{\prime \prime}(x)=0$, $x \in\left[\xi_{k}, \xi_{k+1}\right]$.

If $S^{\prime}(a)=S^{\prime}(b)=0$ then again there are $n+1$ zeros of $S^{\prime}$ in $\left[x_{0}, x_{n}\right]$ and $n$ zeros of $S^{\prime \prime}$ in $\left(x_{0}, x_{n}\right)$. If $S^{\prime \prime}(a)=S^{\prime \prime}(b)=0$ then $S^{\prime \prime}$ has $n$ zeros in $\left[x_{0}, x_{n}\right]$. Using different kind boundary conditions at different endpoints we also arrive at the situation with $S^{\prime \prime}(x)=0, x \in\left[\xi_{k}, \xi_{k+1}\right]$.

Let us make some observations about the situation of $S^{\prime \prime}(x)=0$, $x \in\left[\xi_{k}, \xi_{k+1}\right]$. Then $S$ is at most first degree polynomial on $\left[\xi_{k}, \xi_{k+1}\right]$. If $S$ keeps the sign in $\left[\xi_{k}, x_{k}\right]$ then due to $\int_{x_{k-1}}^{x_{k}} S(x) d x=0$ we have $\eta_{k} \in\left(x_{k-1}, \xi_{k}\right)$ with $S\left(\eta_{k}\right)=0$. We call this case suitable for the left. If $S$ keeps the sign in $\left[x_{k}, \xi_{k+1}\right]$ then $S$ has a zero in $\left(\xi_{k+1}, x_{k+1}\right)$ and this case is called suitable for the right. If, e.g., $k=1$, then $S$ has a zero in $\left(x_{0}, x_{1}\right), S$ keeps the sign in $\left[x_{1}, \xi_{2}\right]$, due to $\int_{x_{1}}^{x_{2}} S(x) d x=0$ there is a zero of $S$ in $\left[\xi_{2}, x_{2}\right)$ and this case is suitable for the right. Similarly, $k=n-1$ (i.e., $k+1=n$ ) is a case suitable for the left.

Consider now the case of $\left[\xi_{k}, \xi_{k+1}\right]$ suitable for the left. The interval $\left[a, \xi_{k}\right]$ contains $k-1$ subintervals $\left[\xi_{1}, \xi_{2}\right], \ldots,\left[\xi_{k-1}, \xi_{k}\right]$. We know that $S\left(\eta_{i}\right)=0$, $\eta_{i} \in\left(x_{i-1}, x_{i}\right), i=1, \ldots, k-1$, and $S\left(\eta_{k}\right)=0, \eta_{k} \in\left(x_{k-1}, \xi_{k}\right]$. We have: 
1) case $S(a)=0$, then $S$ has $k+1$ zeros $a, \eta_{1}, \ldots, \eta_{k}, S^{\prime}$ has $k$ zeros, $S^{\prime \prime}$ has $k-1$ zeros in $\left(a, \xi_{k}\right)$ and $S^{\prime \prime}\left(\xi_{k}\right)=0$;

2) case $S^{\prime}(a)=0$, then $S$ has $k$ zeros $\eta_{1}, \ldots, \eta_{k}, S^{\prime}$ has $k-1$ zeros in $\left(a, \xi_{k}\right)$ and $S^{\prime}(a)=0, S^{\prime \prime}$ has $k-1$ zeros in $\left(a, \xi_{k}\right)$ and $S^{\prime \prime}\left(\xi_{k}\right)=0$;

$3)$ case $S^{\prime \prime}(a)=0$, then $S$ has $k$ zeros in $\left(a, \xi_{k}\right], S^{\prime}$ has $k-1$ zeros, $S^{\prime \prime}$ has $k-2$ zeros in $\left(a, \xi_{k}\right)$ and $S^{\prime \prime}(a)=0, S^{\prime \prime}\left(\xi_{k}\right)=0$.

Anyway, $S^{\prime \prime}$ has $k$ zeros in $k-1$ subintervals and, thus, $S^{\prime \prime}$ is again equal to zero in some of them.

Observe that receiving $S^{\prime \prime}(x)=0, x \in\left[\xi_{k-1}, \xi_{k+1}\right]$, first degree polynomial $S$ on $\left[\xi_{k-1}, \xi_{k+1}\right]$, due to $\int_{x_{k-1}}^{x_{k}} S(x) d x=0$, has a zero in $\left(x_{k-1}, x_{k}\right)$ and keeps the sign in $\left[\xi_{k-1}, x_{k-1}\right]$, consequently, $\left[\xi_{k-1}, \xi_{k}\right]$ is suitable for the left. At the same time, $S$ keeps the sign in $\left[x_{k}, \xi_{k+1}\right]$ and $\left[\xi_{k}, \xi_{k+1}\right]$ is suitable for the right.

Presented reasonings allow to assert that during the process there are always adjacent subintervals $\left[\xi_{j}, \xi_{j+1}\right], \ldots,\left[\xi_{k-1}, \xi_{k}\right]$ where the nullity of $S^{\prime \prime}$ is not yet established, but $\left[\xi_{j-1}, \xi_{j}\right]$ is suitable for the right and $\left[\xi_{k}, \xi_{k+1}\right]$ is suitable for the left which yields that $S^{\prime \prime}$ is equal to zero on one of them. However, it may be as well $j=1$ or $k=n$. The process ends at $S^{\prime \prime}(x)=0, x \in[a, b]$, and then $S(x)=0, x \in[a, b]$, by histopolation and boundary conditions. Naturally, suppose that $n \geq 2$ if we use $S^{\prime \prime}(a)=\alpha$ and $S^{\prime \prime}(b)=\beta$.

\section{Another representation}

Consider the histopolation problem posed in Section 2. A classical representation of cubic spline is the use of $S_{i}=S\left(\xi_{i}\right), M_{i}=S^{\prime \prime}\left(\xi_{i}\right), i=1, \ldots, n$.

Any cubic spline satisfies the internal equations (continuity of $S^{\prime}$ at knots $\xi_{i}$ )

$\frac{\delta_{i-1}}{\delta_{i-1}+\delta_{i}} M_{i-1}+2 M_{i}+\frac{\delta_{i}}{\delta_{i-1}+\delta_{i}} M_{i+1}=6 \frac{\frac{S_{i+1}-S_{i}}{\delta_{i}}-\frac{S_{i}-S_{i-1}}{\delta_{i-1}}}{\delta_{i-1}+\delta_{i}}, \quad i=2, \ldots, n-1$.

For definiteness, add boundary conditions $M_{1}=\alpha$ (first equation) and $M_{n}=\beta$ (last equation). We obtain the system

$$
A M=B S+d,
$$

where $M=\left(M_{1}, \ldots, M_{n}\right), S=\left(S_{1}, \ldots, S_{n}\right)$, first and last rows of $B$ are zero rows, $d=(\alpha, 0, \ldots, 0, \beta)$. The matrix $A$ has diagonal dominance in rows which gives its invertibility. Note that the diagonal dominance of $A$ in rows takes place also in case of other boundary conditions.

Basing on (3.1) we have

$$
\begin{aligned}
S_{i} & =a_{i}-b_{i} \varepsilon_{i}+c_{i} \varepsilon_{i}^{2}-d_{i} \varepsilon_{i}^{3}, \\
S_{i+1} & =a_{i}+b_{i} \eta_{i}+c_{i} \eta_{i}^{2}+d_{i} \eta_{i}^{3} .
\end{aligned}
$$

From them we obtain

$$
a_{i}=\frac{\eta_{i} S_{i}+\varepsilon_{i} S_{i+1}}{\delta_{i}}-c_{i} \varepsilon_{i} \eta_{i}+d_{i}\left(\varepsilon_{i}-\eta_{i}\right) \varepsilon_{i} \eta_{i},
$$




$$
b_{i}=\frac{S_{i+1}-S_{i}}{\delta_{i}}+c_{i}\left(\varepsilon_{i}-\eta_{i}\right)-d_{i}\left(\eta_{i}^{2}-\varepsilon_{i} \eta_{i}+\varepsilon_{i}^{2}\right)
$$

The coefficients $c_{i}$ and $d_{i}$ were expressed via $M_{i}$ and $M_{i+1}$ in Section 3. Using (3.1) the histopolation conditions could be written

$a_{i-1} \eta_{i-1}+\frac{b_{i-1}}{2} \eta_{i-1}^{2}+\frac{c_{i-1}}{3} \eta_{i-1}^{3}+\frac{d_{i-1}}{4} \eta_{i-1}^{4}+a_{i} \varepsilon_{i}-\frac{b_{i}}{2} \varepsilon_{i}^{2}+\frac{c_{i}}{3} \varepsilon_{i}^{3}-\frac{d_{i}}{4} \varepsilon_{i}^{4}=z_{i} h_{i}$

or

$$
\begin{aligned}
& \frac{\eta_{i-1}^{2}}{2 \delta_{i-1}} S_{i-1}+\left(\frac{\varepsilon_{i-1} \eta_{i-1}}{\delta_{i-1}}+\frac{\eta_{i-1}^{2}}{2 \delta_{i-1}}+\frac{\varepsilon_{i} \eta_{i}}{\delta_{i}}+\frac{\varepsilon_{i}^{2}}{2 \delta_{i}}\right) S_{i}+\frac{\varepsilon_{i}^{2}}{2 \delta_{i}} S_{i+1} \\
& -\frac{\eta_{i-1}^{2}}{24 \delta_{i-1}}\left(2 \varepsilon_{i-1}^{2}+4 \varepsilon_{i-1} \eta_{i-1}+\eta_{i-1}^{2}\right) M_{i-1} \\
& -\left(\frac{\eta_{i-1}^{2}}{24 \delta_{i-1}}\left(4 \varepsilon_{i-1}^{2}+4 \varepsilon_{i-1} \eta_{i-1}+\eta_{i-1}^{2}\right)+\frac{\varepsilon_{i}^{2}}{24 \delta_{i}}\left(\varepsilon_{i}^{2}+4 \varepsilon_{i} \eta_{i}+4 \eta_{i}^{2}\right)\right) M_{i} \\
& -\frac{\varepsilon_{i}^{2}}{24 \delta_{i}}\left(\varepsilon_{i}^{2}+4 \varepsilon_{i} \eta_{i}+2 \eta_{i}^{2}\right) M_{i+1}=z_{i} h_{i}, \quad i=2, \ldots, n-1 .
\end{aligned}
$$

Near the boundary we get

$$
\begin{aligned}
& \left(\frac{\eta_{1} h_{1}}{\delta_{1}}+\frac{h_{1}^{2}}{2 \delta_{1}}\right) S_{1}+\frac{h_{1}^{2}}{2 \delta_{1}} S_{2} \\
& -\frac{h_{1}^{2}}{24 \delta_{1}}\left(4 \eta_{1}^{2}+4 \eta_{1} h_{1}+h_{1}^{2}\right) M_{1}-\frac{h_{1}^{2}}{24 \delta_{1}}\left(2 \eta_{1}^{2}+4 \eta_{1} h_{1}+h_{1}^{2}\right) M_{2}=z_{1} h_{1}
\end{aligned}
$$

with the counterpart containing $z_{n} h_{n}$. These equations together form the system

$$
C S=D M+E z .
$$

Note that in matrices $C$ and $D$ the diagonal dominates in rows, $E$ is diagonal matrix with entries $h_{i}$ and $z=\left(z_{1}, \ldots, z_{n}\right)$. Clearly, to construct the cubic spline histopolant it is necessary and sufficient to solve the system (6.2), (6.5). An opportunity to solve it is the following. Take, e.g., a guess value $M^{0}=\left(M_{1}, M_{2}^{0}, \ldots, M_{n-1}^{0}, M_{n}\right), M_{i}^{0}=D_{i} / 2 h_{i}^{3}, i=2, \ldots, n-1$ (note that, in uniform grid case, $D_{i} / 2$ is close to $h^{3} f^{\prime \prime}\left(x_{i}\right)$ if the values $z_{i}$ are determined as in Section 7), then find $S^{0}$ from $C S^{0}=D M^{0}+E z, M^{1}$ from $A M^{1}=B S^{0}+d, S^{1}$ from $C S^{1}=D M^{1}+E z$, in general, the iteration process is $A M^{k}=B S^{k-1}+d$, $C S^{k}=D M^{k}+E z, k=1,2, \ldots$. It may be deduced here also the process

$$
M^{k}=A^{-1} B C^{-1} D M^{k-1}+A^{-1} B C^{-1} E z+A^{-1} d
$$

and the convergence is defined by the spectrum of $A^{-1} B C^{-1} D$. Another opportunity is to take a guess value $S^{0}=\left(S_{1}^{0}, \ldots, S_{n}^{0}\right)$, e.g., $S_{i}^{0}=z_{i}, i=1, \ldots, n$, then find $M^{0}$ from $A M^{0}=B S^{0}+d$, in general, $A M^{k-1}=B S^{k-1}+d$, $C S^{k}=D M^{k-1}+E z, k=1,2, \ldots$. This process could be described as

$$
S^{k}=C^{-1} D A^{-1} B S^{k-1}+C^{-1} D A^{-1} d+C^{-1} E z .
$$


It is immediate to check that the eigenvalues of $C^{-1} D A^{-1} B$ and $A^{-1} B C^{-1} D$ coincide.

We will give examples of spectrum in some particular cases in next section.

Let us consider now the uniform mesh with central spline knots, i.e., $h_{i}=h$, $i=1, \ldots, n, \xi_{i}=\left(x_{i-1}+x_{i}\right) / 2, i=2, \ldots, n-1$. Equation (6.1) is well known in treatments about cubic splines, it should be taken into account that $\delta_{1}=\delta_{n-1}=3 h / 2, \delta_{i}=h, i=2, \ldots, n-2$. Equation $(6.3)$ is

$$
\begin{aligned}
S_{i-1}+6 S_{i}+S_{i+1} & =\frac{h^{2}}{48}\left(7 M_{i-1}+18 M_{i}+7 M_{i+1}\right)+8 z_{i}, \quad i=3, \ldots, n-2, \\
2 S_{1}+19 S_{2}+3 S_{3} & =\frac{h^{2}}{48}\left(34 M_{1}+77 M_{2}+21 M_{3}\right)+24 z_{2}
\end{aligned}
$$

and (6.4) is now

$$
2 S_{1}+S_{2}=\frac{h^{2}}{24}\left(8 M_{1}+7 M_{2}\right)+3 z_{1} .
$$

\section{Numerical tests}

We histopolated the function $f(x)=1 / x^{2}, x \in[-2,-0.1]$, on uniform grid for $n=8$ and central spline knots $\xi_{i}=\left(x_{i-1}+x_{i}\right) / 2, i=2, \ldots, n-1$. Histogram heights were computed as $z_{i}=\frac{1}{h} \int_{x_{i-1}}^{x_{i}} f(x) d x, i=1, \ldots, n$. Resulting histopolants $S$ are given in Figures 2-4.

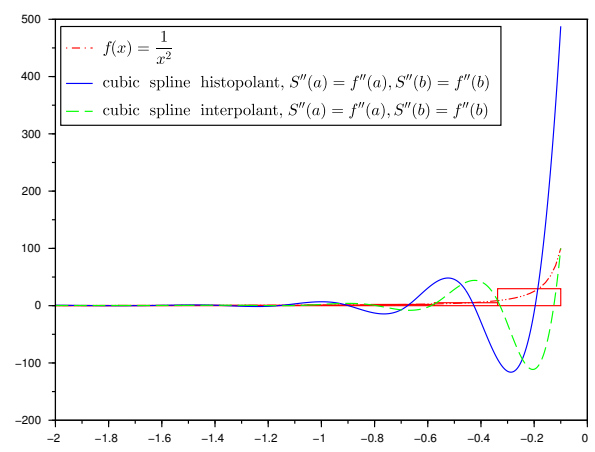

Figure 2. Cubic spline histopolant and interpolant for $n=8$

In Figure 2 the histopolant with boundary conditions $S^{\prime \prime}(a)=f^{\prime \prime}(a)$, $S^{\prime \prime}(b)=f^{\prime \prime}(b)$; in Figure 3 the histopolant with boundary conditions $S^{\prime}(a)=$ $f^{\prime}(a), S^{\prime}(b)=f^{\prime}(b)$; in Figure 4 the histopolant with boundary conditions $S(a)=f(a), S(b)=f(b)$. In comparison also cubic spline interpolants are given satisfying interpolation conditions $S\left(x_{i}\right)=f\left(x_{i}\right), i=0, \ldots, n$.

Considering the representation used in Section 6 we tested the dependence of eigenvalues of matrix $A^{-1} B C^{-1} D$ on grid points $x_{i}$ and spline knots $\xi_{i}$. Again the case $n=8$ is analysed for different meshes. 


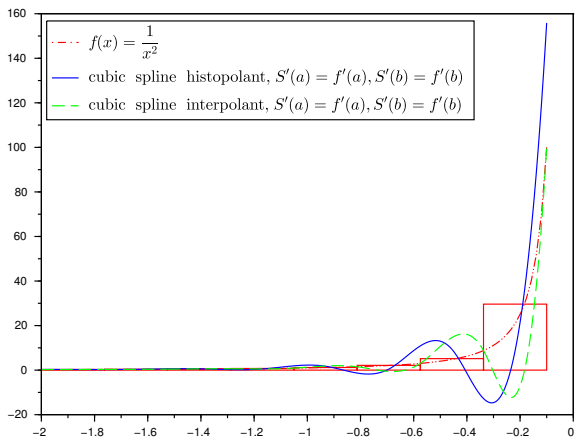

Figure 3. Cubic spline histopolant and interpolant for $n=8$

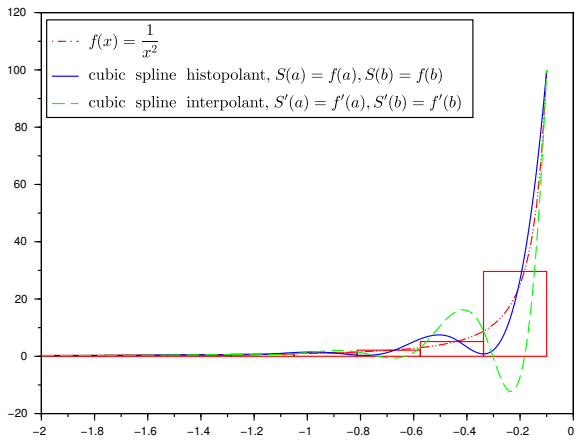

Figure 4. Cubic spline histopolant and interpolant for $n=8$

1) Uniform grid $x_{i}=a+i h, i=0, \ldots, n$, and central spline knots $\xi_{i}=$ $\left(x_{i-1}+x_{i}\right) / 2, i=2, \ldots, n-1$, give the maximal by modulus eigenvalue $\left|\lambda_{\max }\right|=0.271$.

2) For uniform histogram grid $x_{i}=a+i h, i=0, \ldots, n$, spline knots $\xi_{i}=$ $\left(x_{i-1}+x_{i}\right) / 2, i=2,3,6,7, \xi_{4}=0.1 x_{3}+0.9 x_{4}, \xi_{5}=0.9 x_{4}+0.1 x_{5}\left(\xi_{4}\right.$ and $\xi_{5}$ are close to $\left.x_{4}\right)$ it holds $\left|\lambda_{\max }\right|=2.388$.

3) For uniform grid $x_{i}=a+i h, i=0, \ldots, n$, spline knots $\xi_{i}=\left(x_{i-1}+x_{i}\right) / 2$, $i=2,3,6,7, \xi_{4}=0.9 x_{3}+0.1 x_{4}, \xi_{5}=0.1 x_{4}+0.9 x_{5}\left(\xi_{4}\right.$ and $\xi_{5}$ are close to $x_{3}$ and $x_{5}$, respectively) it holds $\left|\lambda_{\max }\right|=0.803$.

4) Take $h=(b-a) / n, h_{i}=0.1 h, i=1,3,5,7, h_{i}=1.9 h, i=2,4,6,8$, and central spline knots $\xi_{i}=\left(x_{i-1}+x_{i}\right) / 2, i=2, \ldots, n-1$, then $\left|\lambda_{\max }\right|=0.241$. 


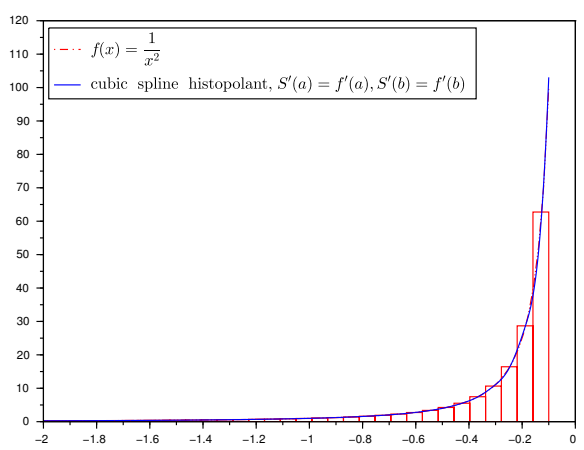

Figure 5. Cubic spline histopolant for $n=32$

\section{Concluding remarks}

Construction of histopolating cubic spline could be done using second derivatives $M_{i}$ and particular integrals $\lambda_{i}, \rho_{i}$. The crucial moment here is the solution of system (4.9). In case of diagonal dominance in the matrix of (4.9) standard methods (e.g., Gaussian elimination) are stable. In absence of diagonal dominance it may be that other methods should be applied. One way to continue is to solve the system determining parameters $\lambda_{i}, \rho_{i}$. Another natural way is to solve system (6.5) where the matrix $C$ has diagonal dominance. An opportunity is to use an iteration process described in Section 6 to determine either second derivatives or spline values and then the others by (6.2) or (6.5) with a matrix having diagonal dominance. We have seen in Section 7 that the convergence may be slow or be absent at all. In return, in the presence of convergence, the calculations at iteration are stable.

Numerical tests with the function $1 / x^{2}$ confirmed the known fact that polynomial splines (at interpolation or histopolation) do not preserve geometrical properties like positivity, monotonicity, convexity. However, increasing the number $n$ of knots, the cubic spline histopolant (and cubic spline interpolant) occurs to have these properties because of the uniform convergence of values, first and second derivatives (see Figure 5). For the cubic spline histopolant this follows from the uniform convergence (see, e.g., [1]) of first, second and third derivatives of interpolating quartic splines in equivalent problem as described in Introduction.

\section{References}

[1] J.H. Ahlberg, E. N. Nilson and J. L. Walsh. The Theory of Splines and Their Applications. Academic Press, New York-London, 1967.

[2] C. de Boor. A Practical Guide to Splines. Springer-Verlag, New York, 2001. https://doi.org/10.1007/978-1-4612-6333-3. 
[3] F.-J. Delvos. Periodic area matching interpolation. In Numerical methods of approximation theory, Vol. 8 (Oberwolfach, 1986), volume 81 of Internat. Schriftenreihe Numer. Math., pp. 54-66, Basel, 1987. Birkhäuser. https://doi.org/10.1007/978-3-0348-6656-9_5.

[4] F.-J. Delvos. Optimal periodic interpolation in the mean. In Numerical methods and approximation theory, III (Niš, 1987), pp. 179-189, Niš, 1988. Univ. Niš.

[5] V. Deputat, P. Oja and D. Saveljeva. Quadratic spline subdomain method for Volterra integral equations. Math. Model. Anal., 10(4):335-344, 2005.

[6] H.P. Dikshit and P. Powar. Area matching interpolation by discrete cubic splines. In Approximation theory and applications (St. John's, Nfld., 1984), volume 133 of Res. Notes in Math., pp. 35-45, Boston, 1985. Pitman.

[7] M. Fischer and P. Oja. Monotonicity preserving rational spline histopolation. J. Comput. Appl. Math., 175(2):195-208, 2005. https://doi.org/10.1016/j.cam.2004.05.009.

[8] M. Fischer, P. Oja and H. Trossmann. Comonotone shape-preserving spline histopolation. J. Comput. Appl. Math., 200(1):127-139, 2007. https://doi.org/10.1016/j.cam.2005.12.010.

[9] H. Hallik. Rational spline histopolation, Ph.D. thesis. University of Tartu, 2015.

[10] A.K.A. Khalifa and J.C. Eilbeck. Collocation with quadratic and cubic splines. IMA J. Numer. Anal., 2(1):111-121, 1982. https://doi.org/10.1093/imanum/2.1.111.

[11] J. Kobza. Spline recurrences for quartic splines. Acta Univ. Palack. Olomuc. Fac. Rerum Natur. Math., 34:75-89, 1995.

[12] J. Kobza. Quartic interpolatory splines. Studia Univ. Babeş-Bolyai Math., 41(3):33-50, 1996.

[13] J. Kobza. Local representations of quartic splines. Acta Univ. Palack. Olomuc. Fac. Rerum Natur. Math., 36:63-78, 1997.

[14] R. Morandi and P. Costantini. Piecewise monotone quadratic histosplines. SIAM J. Sci. Stat. Comput., 10(2):397-406, 1989. https://doi.org/10.1137/0910026.

[15] P. Oja. The rate of convergence of the method of subregions by cubic splines for boundary value problems (in Russian). Tartu Riikl. Ül. Toimetised, 833:59-65, 1988 .

[16] P. Oja. Comonotone adaptive interpolating splines. BIT, 42(4):842-855, 2002. https://doi.org/10.1023/A:1021908705602.

[17] P. Oja and Ü. Pettai. Solution of boundary value problems by cubic splines (in Russian). Tartu Riikl. Ül. Toimetised, 913:44-53, 1990.

[18] P. Oja and A. Reitsekas. Collocation and subdomain methods with quadratic and cubic splines for boundary value problems (in Russian). Eesti NSV Tead. Akad. Toimetised Füüs.-Mat., 36(2):118-128, 1987.

[19] I.J. Schoenberg. Splines and histograms. Internat. Ser. Numer. Math., 21:277327, 1973. https://doi.org/10.1007/978-3-0348-5979-0_13.

[20] R. Siewer. Histopolating splines. J. Comput. Appl. Math., 220(1-2):661-673, 2008. https://doi.org/10.1016/j.cam.2007.09.014. 